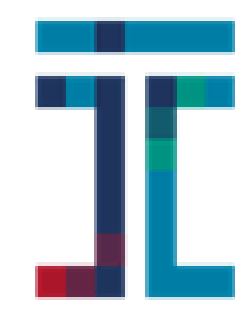

CYPRUS INTERNATIONAL INSTITUTE

FOR ENVIRONMENTAL \& PUBLIC HEALTH

\title{
S3 Questionnaires
}


ID Number

\section{FOOD HABITS QUESTIONNAIRE}

\section{CHILD'S FIRST AND LAST NAME:}

\section{DATE:}

In the last month, how many times have you consumed the following foods: e.g., if you consumed one glass of milk once per day, then you would write 1 in the column times/day and 7 in the column times/week. If it is once per month, then you would write 1 in the column times/month. If you don't remember or you don't know, then simply mark $\sqrt{ }$ in the relevant box (see the example below).

\begin{tabular}{|c|c|c|c|c|c|c|}
\hline \multirow[b]{2}{*}{ Food } & \multirow[b]{2}{*}{ Consumption } & \multicolumn{5}{|c|}{ Frequency } \\
\hline & & Never & Times/Day & $\begin{array}{l}\text { Times/ } \\
\text { Week }\end{array}$ & $\begin{array}{l}\text { Times/ } \\
\text { Month }\end{array}$ & $\begin{array}{l}\text { I don't } \\
\text { remember/ } \\
\text { I don't } \\
\text { know }\end{array}$ \\
\hline \multirow{2}{*}{$\begin{array}{l}\text { Milk (glass of water, } \\
250 \mathrm{ml})\end{array}$} & 1 glass/day & & 1 & 7 & & \\
\hline & 2 glasses/day & & & & 2 & \\
\hline $\begin{array}{c}\text { Yogurt, Full } \\
\text { (Packaging 200g) }\end{array}$ & 3 servings/ week & & & 3 & & \\
\hline \multirow{3}{*}{$\begin{array}{c}\text { Meat } \\
\text { (portion=90gr) }\end{array}$} & $\begin{array}{c}2 \mu \varepsilon \rho i \delta \varepsilon \varepsilon / 1 \eta \mu \varepsilon \dot{\rho} \rho \alpha / \\
\varepsilon \beta \delta \circ \mu \alpha \dot{\delta} \delta \alpha\end{array}$ & & 2 & 1 & & \\
\hline & 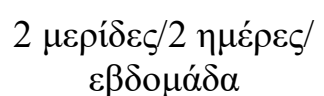 & & 2 & 2 & & \\
\hline & Поте́ & $\sqrt{ }$ & & & & \\
\hline Bread $($ slice $=30 \mathrm{gr})$ & 2 slices $/ \eta \mu \varepsilon ́ \rho \alpha$ & & 2 & 7 & & \\
\hline $\begin{array}{c}\text { Cheese } \\
\text { (portion=30gr) }\end{array}$ & 2 portions/week & & & 2 & & \\
\hline \multirow{2}{*}{$\begin{array}{l}\text { Boiled Vegetables, } \\
\text { e.g. cauliflower etc. } \\
\text { ( } 1 / 2 \text { cup })\end{array}$} & 1 cup/day & & $2(1 \times 2)$ & 7 & & \\
\hline & 2 cups/ week & & & $4(2 \times 2)$ & & \\
\hline $\begin{array}{l}\text { Legumes } \\
(1 / 2 \text { cup })\end{array}$ & 3 cups/week & & & & $6(3 \times 2)$ & \\
\hline Fruits (number) & 3 fruits/day & & 3 & 7 & & \\
\hline
\end{tabular}




\section{QUESTIONNAIRE}

\begin{tabular}{|c|c|c|c|c|c|}
\hline \multirow{2}{*}{ FOOD } & \multicolumn{4}{|c|}{ FREQUENCY } \\
\cline { 2 - 5 } & Never & $\begin{array}{c}\text { Times/ } \\
\text { Day }\end{array}$ & $\begin{array}{c}\text { Times/ } \\
\text { Week }\end{array}$ & $\begin{array}{c}\text { Times/ } \\
\text { Month }\end{array}$ & $\begin{array}{c}\text { I don't } \\
\text { remember/ } \\
\text { I don't } \\
\text { know }\end{array}$ \\
\hline \hline
\end{tabular}

1. Milk (portion=size of glass of water, $250 \mathrm{ml}$ )

\begin{tabular}{|l|l|l|l|l|l|}
\hline a. Fresh whole & & & & & \\
\hline b. Fresh low-fat & & & & & \\
\hline c. Fresh fat-free & & & & & \\
\hline d. Condensed whole & & & & & \\
\hline e. Condensed low-fat & & & & & \\
\hline f. Condensed fat-free & & & & & \\
\hline g. Sweetened & & & & & \\
\hline h. Chocolate & & & & & \\
\hline \hline
\end{tabular}

2. Yogurt (plain or with fruits) (small package of 200g)

\begin{tabular}{|l|l|l|l|l|l|}
\hline \hline a. Full & & & & & \\
\hline b. Low-fat & & & & & \\
\hline c. Fat-free & & & & & \\
\hline
\end{tabular}

3. Cheese (portions are about the size of a matchbox, portion=30gr)

a. Parmesan, Graviera, Kasseri, Cheddar

b. Feta, Halloumi, Fresh Anari, Edam

c. Soft or Cream Cheeses and Spreads (e.g.,

Cottage, Philadelphia, La vache qui rit)

Is the cheese that you usually eat low in fat? (Mark $\sqrt{ }$ in the corresponding box)

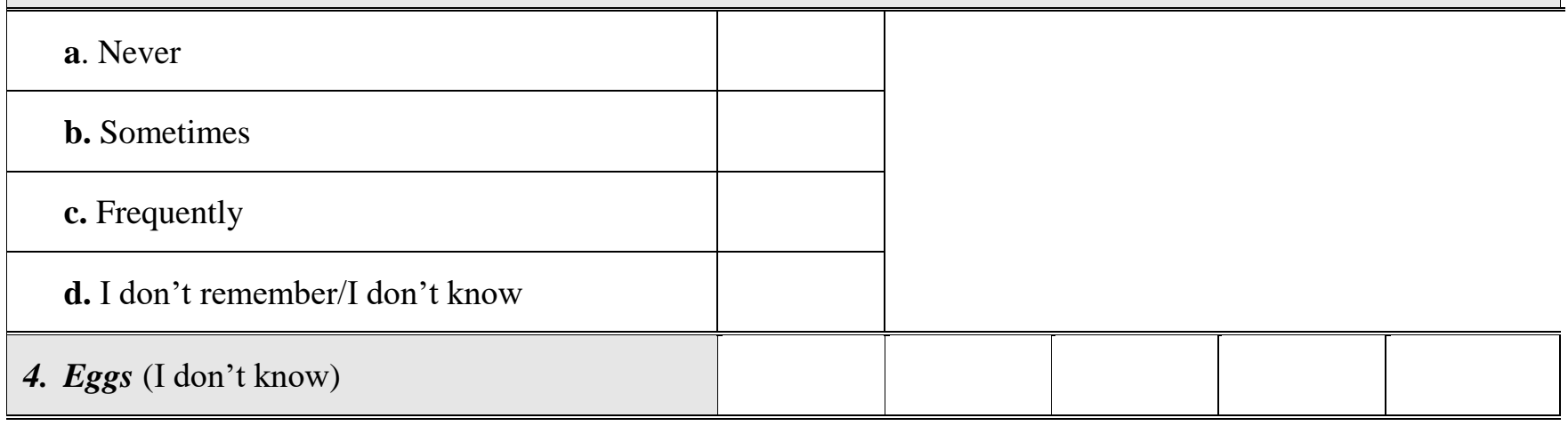




\begin{tabular}{|c|c|c|c|c|c|}
\hline \multirow[b]{2}{*}{ FOOD } & \multicolumn{5}{|c|}{ FREQUENCY } \\
\hline & Never & $\begin{array}{l}\text { Times/ } \\
\text { Day }\end{array}$ & $\begin{array}{l}\text { Times/ } \\
\text { Week }\end{array}$ & $\begin{array}{l}\text { Times/ } \\
\text { Month }\end{array}$ & $\begin{array}{c}\text { I don't } \\
\text { remember/ } \\
\text { I don't } \\
\text { know } \\
\end{array}$ \\
\hline \multicolumn{6}{|c|}{ 5. Morning cereals (medium-sized bowl) } \\
\hline \multicolumn{6}{|c|}{$\begin{array}{l}\text { a. Cornflakes, Rice-Krispies, other cereals low } \\
\text { in fiber }\end{array}$} \\
\hline \multicolumn{6}{|c|}{$\begin{array}{l}\text { b. All-Bran, Fruit 'n' Fiber, Muesli, other } \\
\text { cereals high in fiber }\end{array}$} \\
\hline \multicolumn{6}{|c|}{$\begin{array}{l}\text { c. chocolate or sweetened, e.g.,. Coco-Pops, } \\
\text { Frosties }\end{array}$} \\
\hline \multicolumn{6}{|c|}{ 6. Bread, buns, loaf, pita, toast, crouttons $(\varphi \varepsilon ́ \tau \alpha /$ slice $=30 \gamma \rho)$} \\
\hline \multicolumn{6}{|l|}{ a. White } \\
\hline \multicolumn{6}{|l|}{ b. Village bread/Simit } \\
\hline \multicolumn{6}{|l|}{ c. Black } \\
\hline d. Wheat/Multi-Seeds & & & & & \\
\hline
\end{tabular}

7. Wheat (portion $=1 / 2$ cup $)$

\begin{tabular}{|l|l|l|l|l|l|}
\hline \hline a. Rice & & & & & \\
\hline b. Bulgur & & & & & \\
\hline c. Trahana & & & & & \\
\hline \hline
\end{tabular}

\section{Pasta}

a. Pasta, Orzo (porion= $1 / 2$ cup)

b. Stuffed Pasta (e.g., Ravioli, Tortellini)

(portion $=1 / 2$ cup)

c. Pastitsio (one medium-sized piece)

(number)

\section{Potatoes}

a. Fried (portion $=20-25$ potatoes)

b. Boiled (portion $=2$ small potatoes $-90 \mathrm{gr}$ )

c. Baked (portion $=2$ small potatoes -90 gr)

d. Puree (portion $=1 / 2$ cup)

10. Legumes e.g.,. lentils, beans (portion $=1 / 2$ cup) 


\begin{tabular}{|c|c|c|c|c|c|}
\hline \multirow{2}{*}{ FOOD } & \multicolumn{4}{|c|}{ FREQUENCY } \\
\cline { 2 - 6 } & & Never & $\begin{array}{c}\text { Times/ } \\
\text { Day }\end{array}$ & $\begin{array}{c}\text { Times/ } \\
\text { Week }\end{array}$ & $\begin{array}{c}\text { Times/ } \\
\text { Month }\end{array}$ \\
$\begin{array}{c}\text { I domember/t } \\
\text { I don't } \\
\text { know }\end{array}$ \\
\hline \hline
\end{tabular}

\section{Meat, Meat and fish items}

a. Meat or minced meat: pork (e.g., steak, meatballs, souvlaki, souvla) (portion=90gr)

b. Meat: lamb/goat (e.g., souvla, kleftiko) (portion=90gr)

c. Meat or minced meat: beef (e.g., steak, hamburger) (portion=90gr)

d. Chicken or rabbit (portion=90gr)

e. Liver (portion=90gr)

f. Sausages (portion=1 piece) (accept the times that are used specifically for sandwiches)

i. Salami, bacon

ii. Lountza, ham

g. Sausages (portion=1 Frankfurt Sausage) (except those used in loukanokopites)

h. Canned meat (e.g., ZWAN) (portion=120gr)

i. Canned fish (e.g., tuna) (portion=120gr)

j. fresh or frozen fish, seafood (calamari, octopus, etc.) (portion=90gr)

\section{Fruits and Vegetables}

a. Raw vegetables, salads (portion=1 cup)

b. Cooked vegetables: greens, beans, cauliflower, etc. (portion $=1 / 2$ cup)

c. Fresh fruits (portion=1 apple, 1 medium cluster of grapes, 15 cherries, 2 apricots, 1 medium-sized slice of watermelon)

d. Fresh juice from fresh fruits or vegetables (glass of water, $250 \mathrm{ml}$ )

13. Oils, Spreads, and Olives (which are used in cooking, served to compliment the main dish, and/or as bread spreads)

a. Olive oil (tablespoon)

b. Seed oil, Sunflower oil, etc. (tablespoon)

c. Margarine (teaspoon)

d. Low-fat margarine (e.g., Flora

light/Vitalite) (teaspoon)

e. Butter (teaspoon)

f. Mayonnaise (teaspoon)

g. Olives (number) 


\begin{tabular}{|c|c|c|c|c|c|}
\hline \multirow[b]{2}{*}{ FOOD } & \multicolumn{5}{|c|}{ FREQUENCY } \\
\hline & Never & $\begin{array}{l}\text { Times/ } \\
\text { Day }\end{array}$ & $\begin{array}{l}\text { Times/ } \\
\text { Week }\end{array}$ & $\begin{array}{l}\text { Times/ } \\
\text { Month }\end{array}$ & $\begin{array}{c}\text { I don't } \\
\text { remember/ } \\
\text { I don't } \\
\text { know } \\
\end{array}$ \\
\hline \multicolumn{6}{|l|}{ 14. Sugar, honey, marmalade, etc. } \\
\hline \multicolumn{6}{|l|}{ a. Sugar (teaspoon) } \\
\hline \multicolumn{6}{|l|}{ b. Honey (teaspoon) } \\
\hline \multicolumn{6}{|l|}{ c. Marmalade (etc.) } \\
\hline \multicolumn{6}{|l|}{ d. Fruit Compote (portion=1/2 cup) } \\
\hline \multicolumn{6}{|l|}{ 15. Chocolates, biscuits, ice-cream, sweets, cake } \\
\hline \multicolumn{6}{|l|}{ a. Merenda e.g., Nutella (teaspoon) } \\
\hline \multicolumn{6}{|l|}{$\begin{array}{l}\text { b. Chocolates (e.g., one chocolate } \\
\text { ION=45gr) List ....................................... }\end{array}$} \\
\hline \multicolumn{6}{|l|}{ 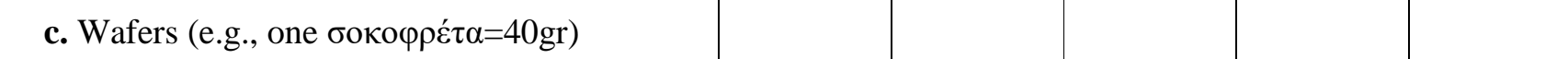 } \\
\hline \multicolumn{6}{|l|}{ d. Cookies (portion=1 cookie) } \\
\hline \multicolumn{6}{|l|}{ e. Ice-cream (portion=1 scoop) } \\
\hline \multicolumn{6}{|l|}{$\begin{array}{l}\text { f. Creams (e.g., pudding, mousse) (1 medium- } \\
\text { sized bowl) }\end{array}$} \\
\hline $\begin{array}{l}\text { g. Cake, tarts, pie, sweets (1 medium-sized } \\
\text { piece) }\end{array}$ & & & & & \\
\hline
\end{tabular}

\section{Snacks and meals}

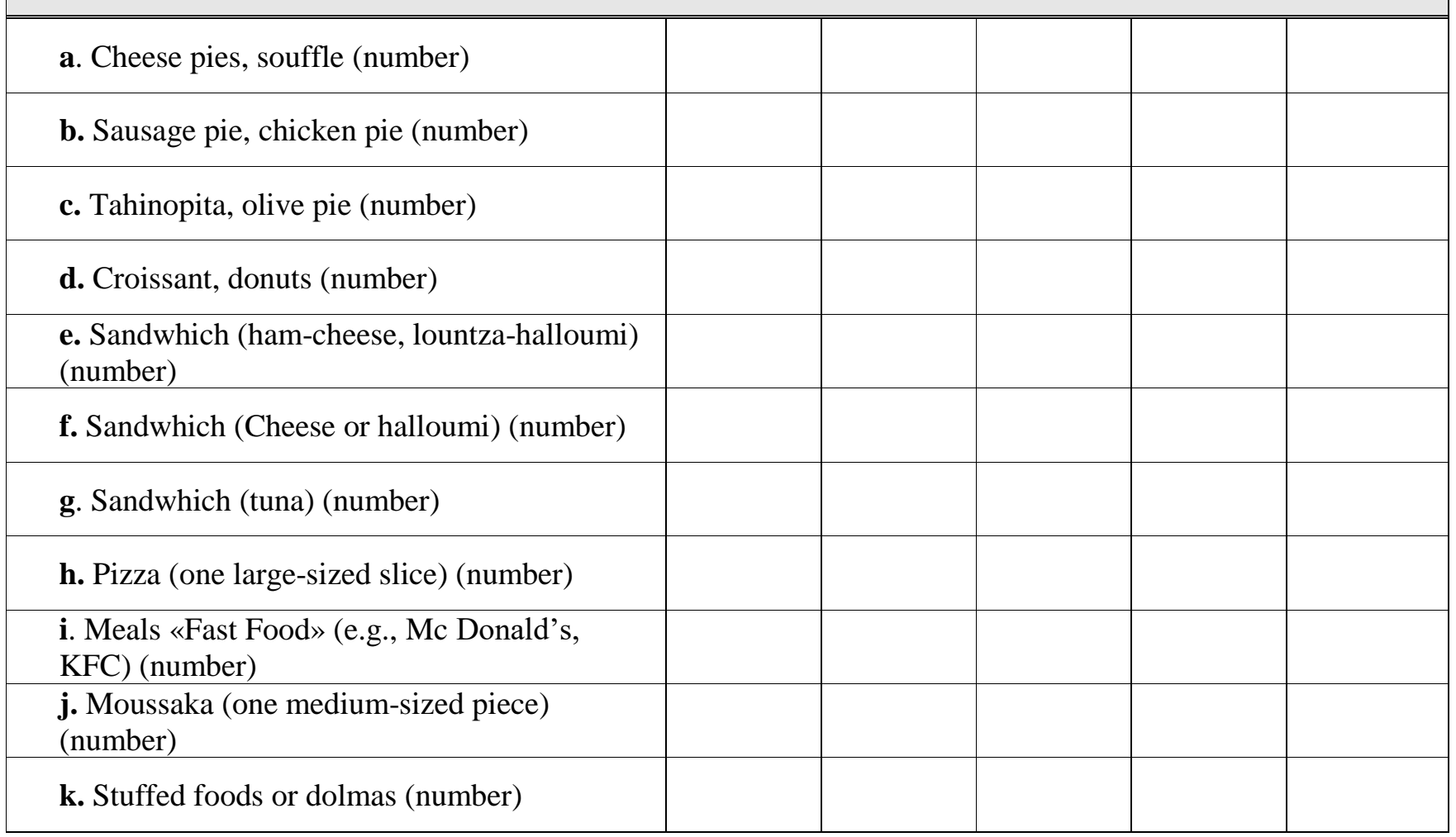




\begin{tabular}{|c|c|c|c|c|c|}
\hline \multirow[b]{2}{*}{ FOOD } & \multicolumn{5}{|c|}{ FREQUENCY } \\
\hline & Never & $\begin{array}{l}\text { Times/ } \\
\text { Day }\end{array}$ & $\begin{array}{l}\text { Times/ } \\
\text { Week }\end{array}$ & $\begin{array}{l}\text { Times/ } \\
\text { Month }\end{array}$ & $\begin{array}{l}\text { I don't } \\
\text { remember/ } \\
\text { I don't } \\
\text { know }\end{array}$ \\
\hline \multicolumn{6}{|l|}{ 17. Crisps, potato chips, popcorn (smalls bags) } \\
\hline \multicolumn{6}{|l|}{ 18. Nuts (small coffee cups) } \\
\hline \multicolumn{6}{|l|}{ 19. Drinks and Coffee } \\
\hline \multicolumn{6}{|l|}{ a. Water (glasses) } \\
\hline \multicolumn{6}{|l|}{ b. Soda (Cans, 330cc) } \\
\hline \multicolumn{6}{|l|}{ i. Soda with sugar } \\
\hline \multicolumn{6}{|l|}{ ii. Sugar-free soda (diet) } \\
\hline \multicolumn{6}{|l|}{ c. Juice (1 box/1 glass $250 \mathrm{ml}$ ) } \\
\hline \multicolumn{6}{|l|}{$\begin{array}{l}\text { i. Concentrated juices (e.g., Lanitis, KEAN } \\
\text { etc.) }\end{array}$} \\
\hline \multicolumn{6}{|l|}{ ii. Fruit juices (e.g., Keanita) } \\
\hline \multicolumn{6}{|l|}{ d. Other drinks (1 box/bottle) } \\
\hline \multicolumn{6}{|l|}{ i. Isotonic (e.g.,. Lucozade, Gatorade) } \\
\hline ii. Energy drinks (e.g., Redbull, Shark) & & & & & \\
\hline
\end{tabular}

\section{Supplements}

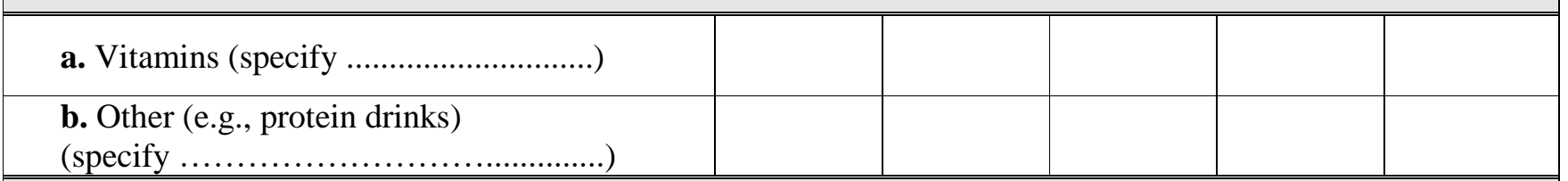

\section{Other foods}

a.

b.

Thank you. 


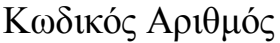

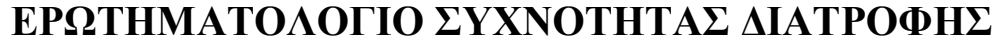

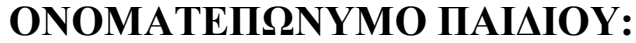

\section{HMEPOMHNIA:}

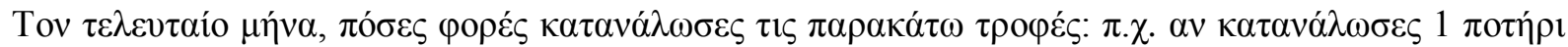

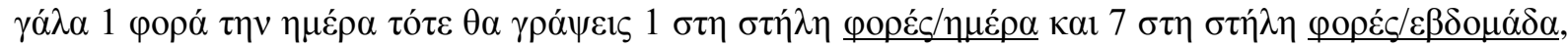

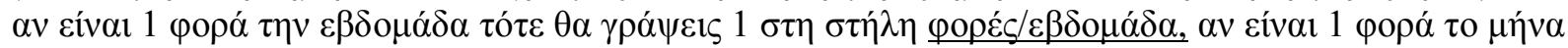

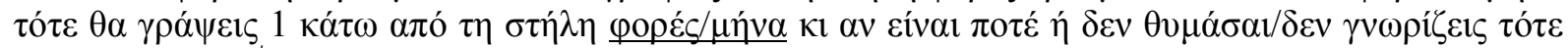

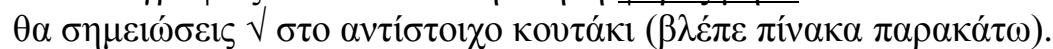

\begin{tabular}{|c|c|c|c|c|c|c|}
\hline \multirow[b]{2}{*}{ Тро́фцца } & \multirow[b]{2}{*}{ 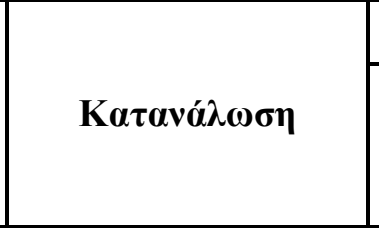 } & \multicolumn{5}{|c|}{$\Sigma v \chi v o ́ \tau \eta \tau \alpha$} \\
\hline & & Поте́ & 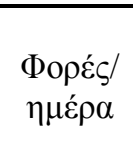 & $\begin{array}{c}\text { Форе́́)/ } \\
\varepsilon \beta \delta \circ \mu \alpha \dot{\delta} \delta \alpha\end{array}$ & 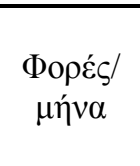 & $\begin{array}{c}\Delta \varepsilon v \\
\theta v \mu \alpha \dot{\mu} \mu \alpha / \\
\Delta \varepsilon v \\
\gamma \nu \omega \rho i ́ \zeta \omega\end{array}$ \\
\hline \multirow{2}{*}{ 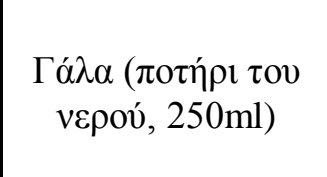 } & 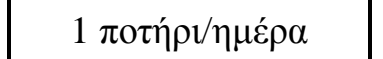 & & 1 & 7 & & \\
\hline & 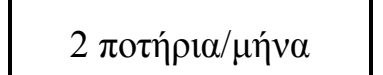 & & & & 2 & \\
\hline 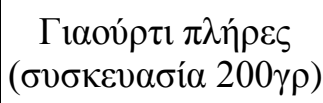 & $\begin{array}{c}3 \kappa \varepsilon \sigma \varepsilon \delta \alpha \dot{\alpha} \kappa \alpha / \\
\varepsilon \beta \delta \circ \mu \alpha \dot{\delta} \delta \alpha\end{array}$ & & & 3 & & \\
\hline \multirow{3}{*}{ 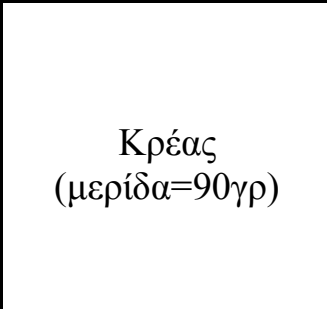 } & 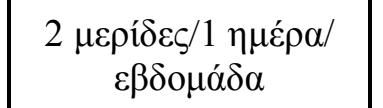 & & 2 & 1 & & \\
\hline & 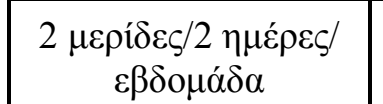 & & 2 & 2 & & \\
\hline & По $\tau \dot{\varepsilon}$ & $\sqrt{ }$ & & & & \\
\hline 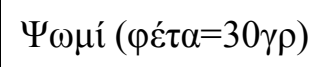 & 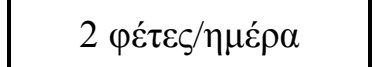 & & 2 & 7 & & \\
\hline $\begin{array}{c}\text { Tvpí } \\
(\mu \varepsilon \rho i ́ \delta \alpha=30 \gamma \rho)\end{array}$ & 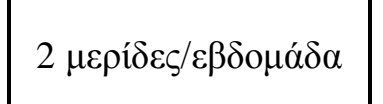 & & & 2 & & \\
\hline \multirow{2}{*}{ 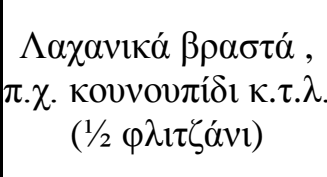 } & 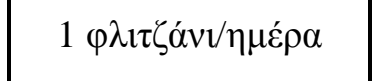 & & $2(1 \times 2)$ & 7 & & \\
\hline & 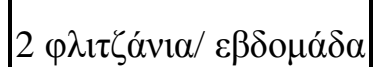 & & & $4(2 \times 2)$ & & \\
\hline 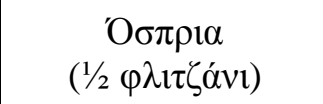 & $3 \varphi \lambda \imath \tau \zeta \alpha ́ v 1 \alpha / \mu \eta ́ v \alpha$ & & & & $6(3 \times 2)$ & \\
\hline$\Phi \rho o v ́ \tau \alpha(\alpha \rho \imath \theta \mu o ́ \varsigma)$ & $3 \varphi \rho o v ́ \tau \alpha / \eta \mu \varepsilon ́ \rho \alpha$ & & 3 & 7 & & \\
\hline
\end{tabular}




\section{ЕРЛТНМАТОАОГІО}

\begin{tabular}{|c|c|c|c|c|c|}
\hline \multirow[b]{2}{*}{ ТРОФІМА } & \multicolumn{5}{|c|}{ ¿YXNOTHTA } \\
\hline & Поте́ & 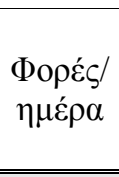 & $\begin{array}{c}\text { Форє́́/ } \\
\varepsilon \beta \delta о \mu \alpha \dot{\delta} \delta \alpha\end{array}$ & $\begin{array}{l}\text { Форв́́/ } \\
\mu \eta ́ v \alpha\end{array}$ & $\begin{array}{c}\Delta \varepsilon v \\
\theta v \mu \alpha ́ \alpha \alpha / \\
\Delta \varepsilon v \\
\gamma v \omega \rho i \zeta \zeta \omega \\
\end{array}$ \\
\hline \multicolumn{6}{|c|}{ 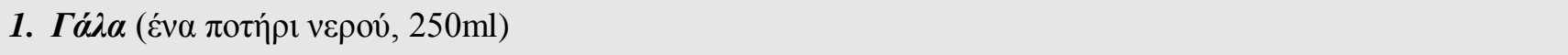 } \\
\hline \multicolumn{6}{|l|}{ 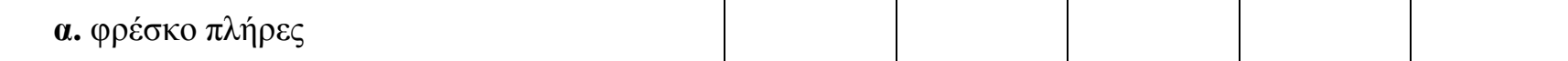 } \\
\hline \multicolumn{6}{|l|}{ 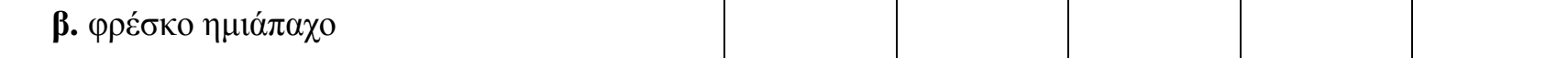 } \\
\hline \multicolumn{6}{|l|}{ 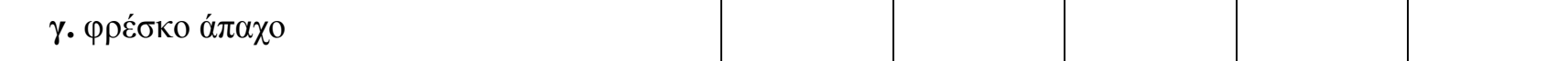 } \\
\hline \multicolumn{6}{|l|}{ 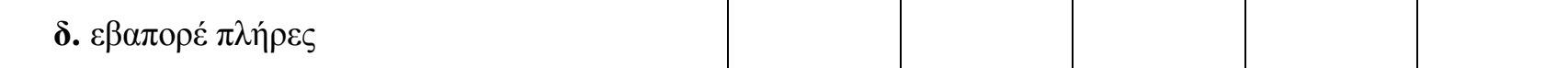 } \\
\hline \multicolumn{6}{|l|}{ 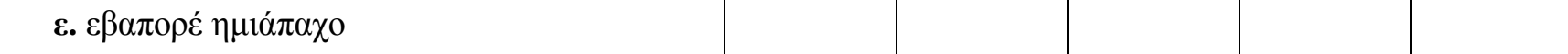 } \\
\hline \multicolumn{6}{|l|}{ 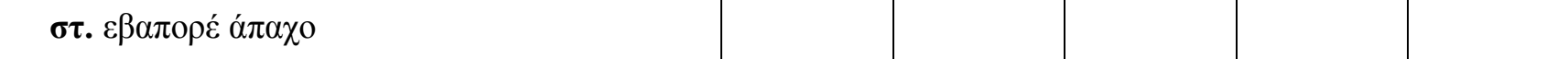 } \\
\hline \multicolumn{6}{|l|}{$\zeta . \zeta \alpha \chi \alpha \rho \circ \chi_{\chi}$} \\
\hline 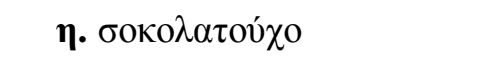 & & & & & \\
\hline
\end{tabular}

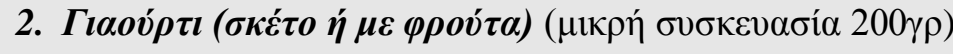

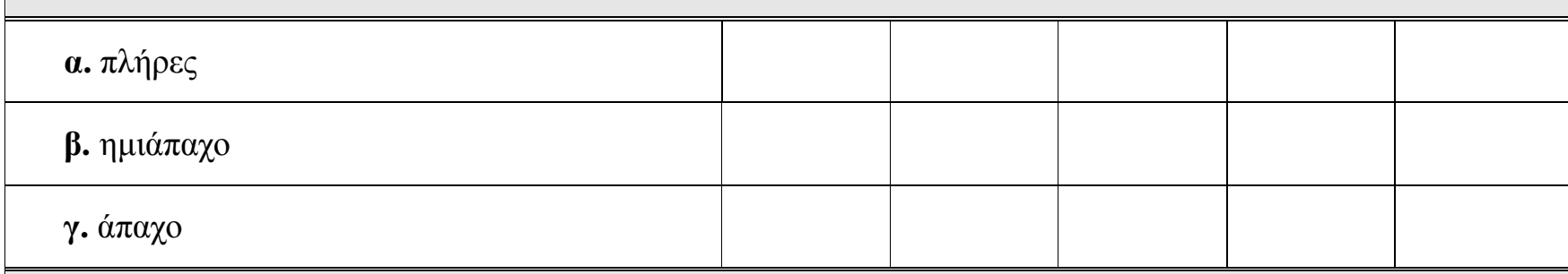

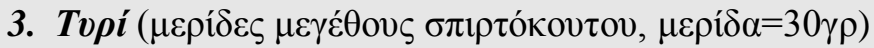

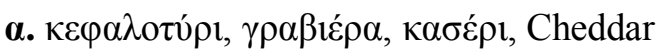

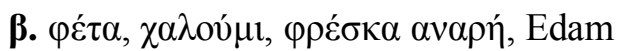

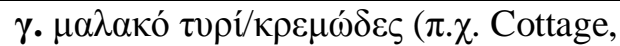

Philadelphia, La vache qui rit)

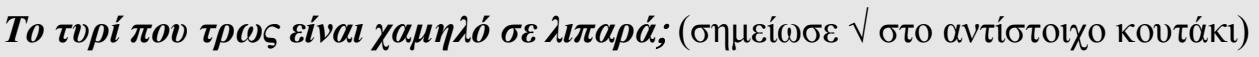

\begin{tabular}{|c|}
\hline 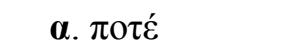 \\
\hline 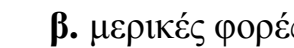 \\
\hline$\gamma . \sigma v v \eta ́ \theta \omega \varsigma / \pi \alpha ́ v$ \\
\hline 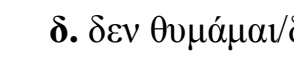 \\
\hline 4. $A v \gamma \alpha \dot{\alpha}(\alpha \rho \imath \theta \mu o ́ \varsigma)$ \\
\hline
\end{tabular}




\begin{tabular}{|c|c|c|c|c|c|}
\hline \multirow[b]{2}{*}{ ТРОФІМА } & \multicolumn{5}{|c|}{ 玉YXNOTHTA } \\
\hline & Поте́ & 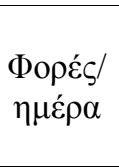 & 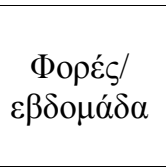 & $\begin{array}{l}\text { Форе́ } / / \\
\mu \eta ́ v \alpha\end{array}$ & 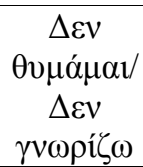 \\
\hline \multicolumn{6}{|l|}{ 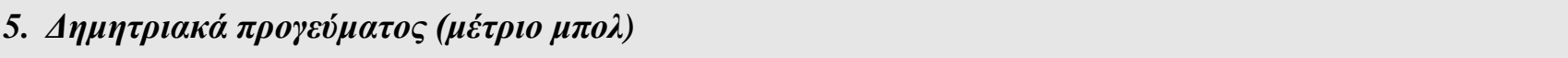 } \\
\hline \multicolumn{6}{|l|}{ 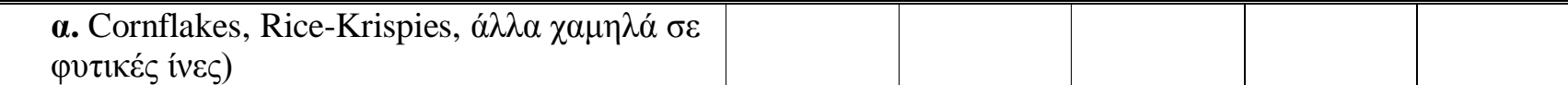 } \\
\hline \multicolumn{6}{|l|}{ 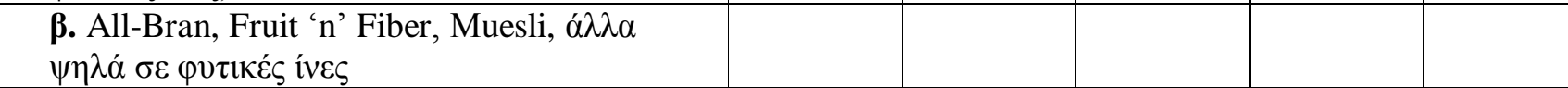 } \\
\hline \multicolumn{6}{|l|}{$\begin{array}{l}\gamma \cdot \sigma o \kappa o \lambda \alpha \tau o v ́ \chi \alpha, \zeta \alpha \chi \alpha \rho o v ́ \chi \alpha, \pi \cdot \chi . \text { Coco-Pops, } \\
\text { Frosties }\end{array}$} \\
\hline \multicolumn{6}{|c|}{ 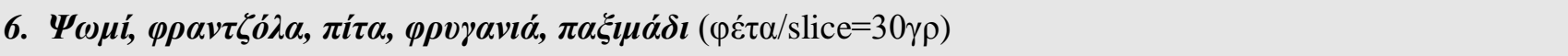 } \\
\hline \multicolumn{6}{|l|}{ 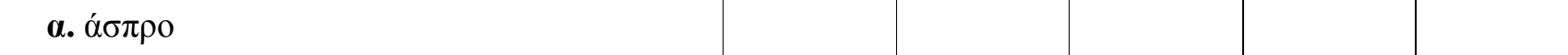 } \\
\hline \multicolumn{6}{|l|}{ 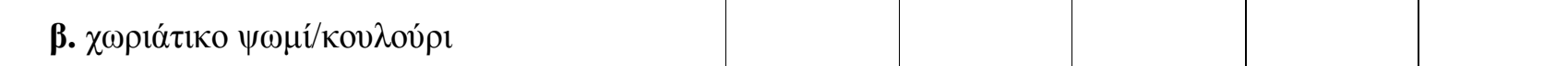 } \\
\hline \multicolumn{6}{|l|}{$\gamma \cdot \mu \alpha u ́ \rho o$} \\
\hline \multicolumn{6}{|l|}{ 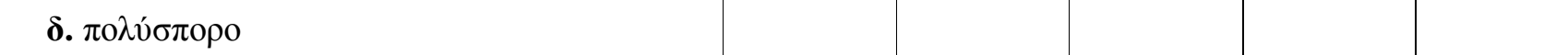 } \\
\hline \multicolumn{6}{|l|}{ 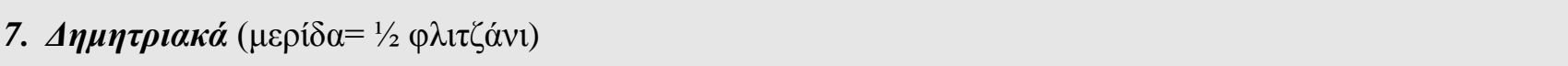 } \\
\hline \multicolumn{6}{|l|}{ a. $\rho v ́ \zeta ı$} \\
\hline \multicolumn{6}{|l|}{ 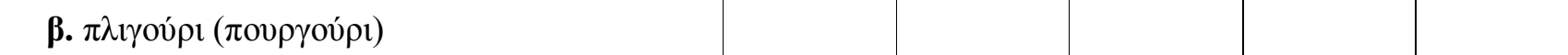 } \\
\hline \multicolumn{6}{|l|}{$\gamma \cdot \tau \rho \alpha \chi \alpha \nu \alpha ́ \varsigma$} \\
\hline \multicolumn{6}{|l|}{ 8. Zvнарıка́ } \\
\hline \multicolumn{6}{|l|}{ 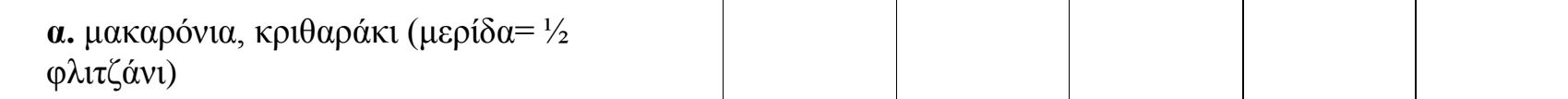 } \\
\hline \multicolumn{6}{|l|}{ 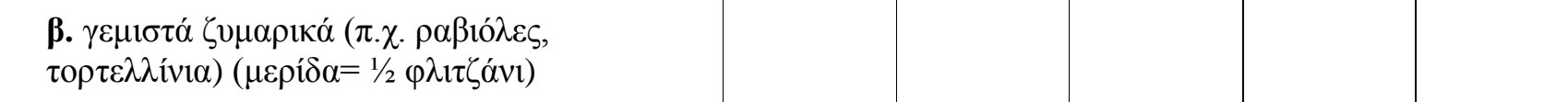 } \\
\hline \multicolumn{6}{|l|}{ 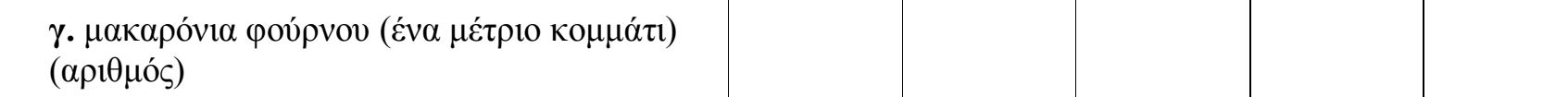 } \\
\hline \multicolumn{6}{|l|}{ 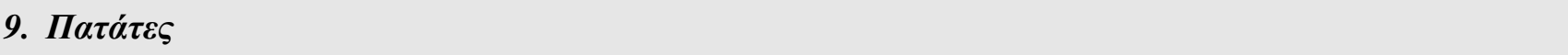 } \\
\hline \multicolumn{6}{|l|}{ 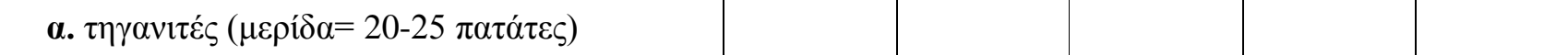 } \\
\hline \multicolumn{6}{|l|}{ 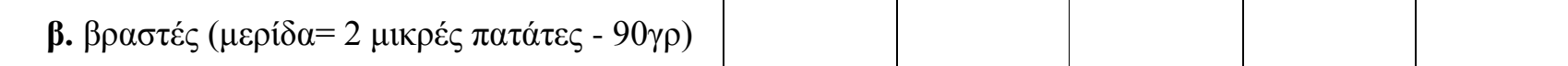 } \\
\hline \multicolumn{6}{|l|}{ 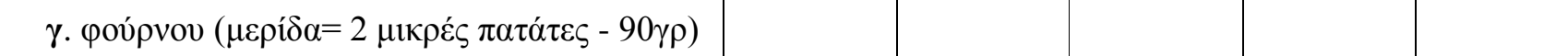 } \\
\hline \multicolumn{6}{|l|}{ 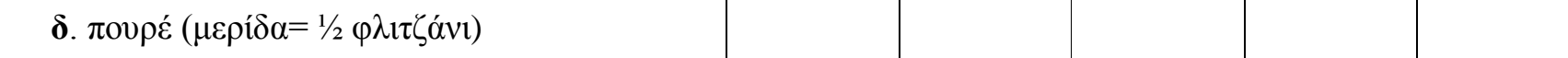 } \\
\hline 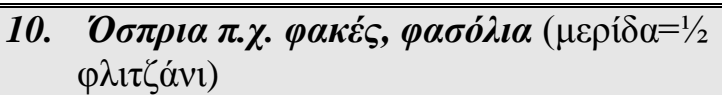 & & & & & \\
\hline
\end{tabular}


¿YXNOTHTA

TPОФIMA

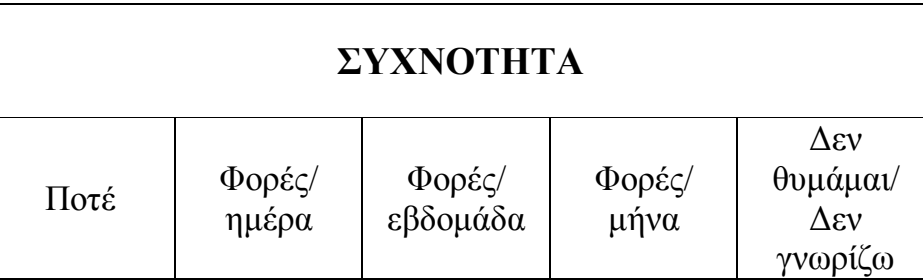

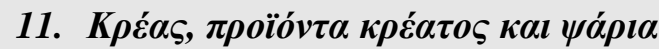

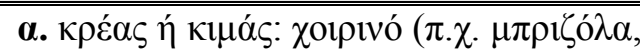

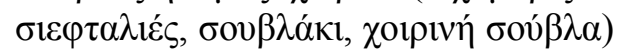
$(\mu \varepsilon \rho i \delta \alpha=90 \gamma \rho)$

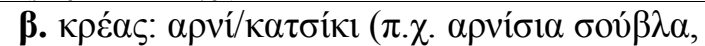

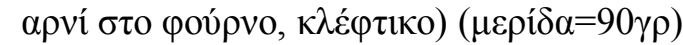

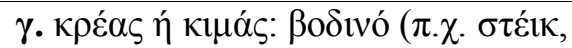
hamburger) $(\mu \varepsilon \rho i ́ \delta \alpha=90 \gamma \rho)$

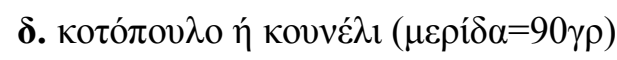

ع. $\sigma v \kappa \omega ́ \tau \imath ~(\mu \varepsilon \rho i ́ \delta \alpha=90 \gamma \rho)$

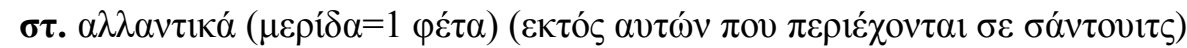

i. $\sigma \alpha \lambda \alpha \dot{\mu} \mu 1, \mu \pi \varepsilon ́ 1 \kappa o v, \mu о v \rho \tau \alpha \tau \varepsilon \dot{\lambda} \alpha$

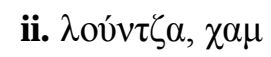

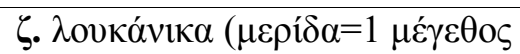

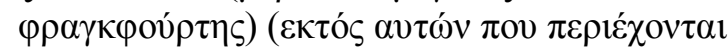

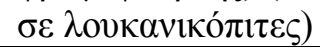

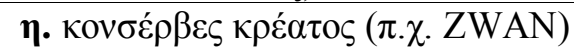
$(\mu \varepsilon \rho i ́ \delta \alpha=120 \gamma \rho)$

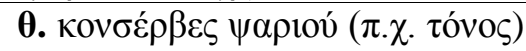
$(\mu \varepsilon \rho i ́ \delta \alpha=120 \gamma \rho)$

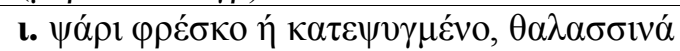

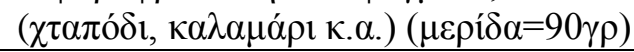

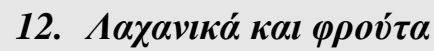

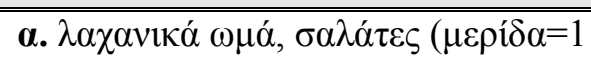
$\varphi \lambda \imath \tau \zeta \dot{\alpha} v 1)$

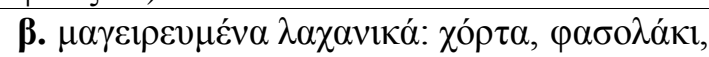

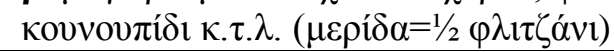

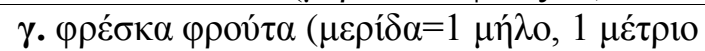

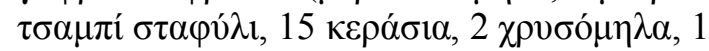

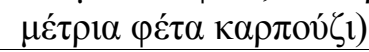

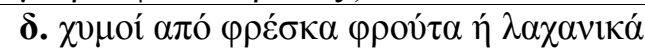

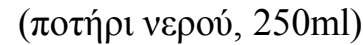

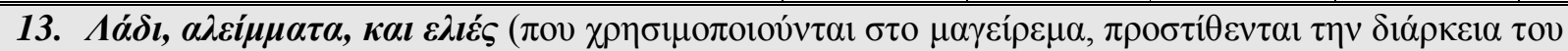

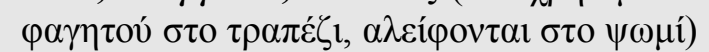

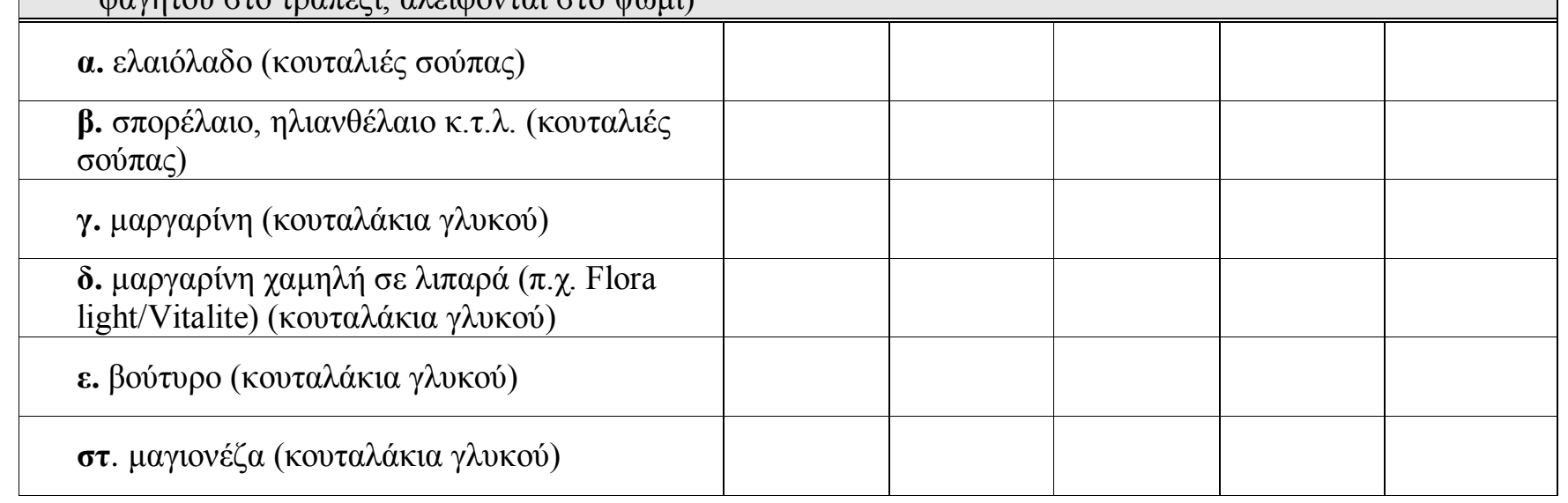




\begin{tabular}{|c|c|c|c|c|c|}
\hline \multirow[b]{2}{*}{ ТРОФIМА } & \multicolumn{5}{|c|}{ 乏YXNOTHTA } \\
\hline & Поте́ & 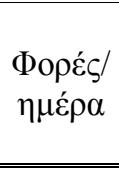 & 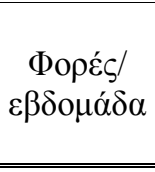 & 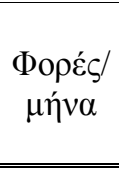 & $\begin{array}{c}\Delta \varepsilon v \\
\theta v \mu \alpha ́ \mu \alpha \mathrm{l} \\
\Delta \varepsilon v \\
\gamma \nu \omega \rho i ́ \zeta \omega \\
\end{array}$ \\
\hline \multicolumn{6}{|l|}{$\zeta . ~ \varepsilon \lambda 1 \varepsilon ́ \varsigma(\alpha \rho t \theta \mu o ́ \varsigma)$} \\
\hline \multicolumn{6}{|l|}{ 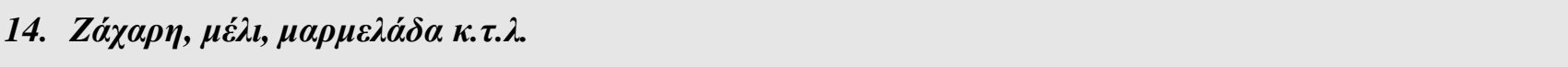 } \\
\hline \multicolumn{6}{|l|}{ 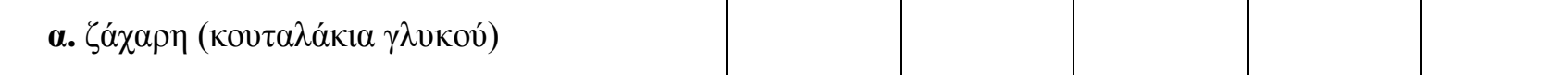 } \\
\hline \multicolumn{6}{|l|}{ 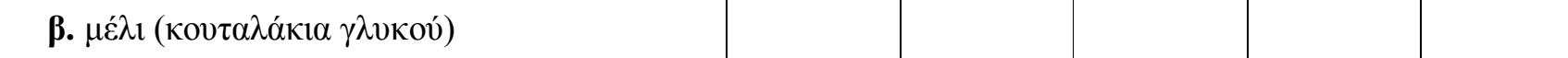 } \\
\hline \multicolumn{6}{|l|}{ 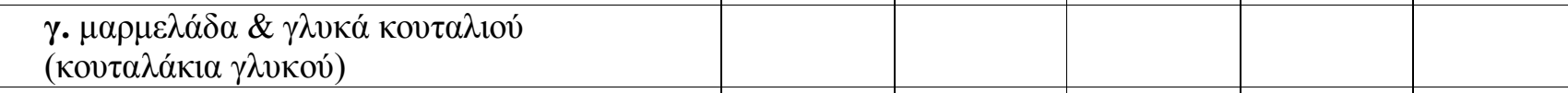 } \\
\hline 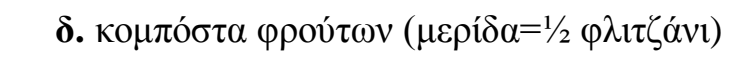 & & & & & \\
\hline
\end{tabular}

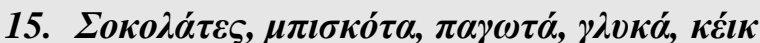

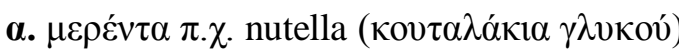

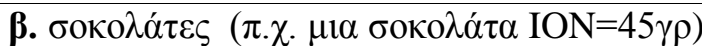
$\pi \rho \circ \sigma \delta$ เó $\rho, \sigma \varepsilon$

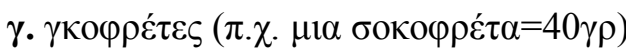

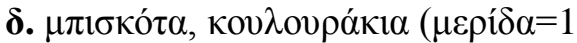

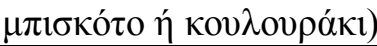

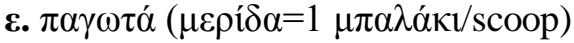

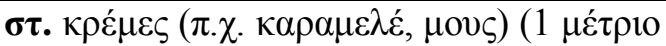
$\mu \pi \mathrm{\partial} \lambda)$

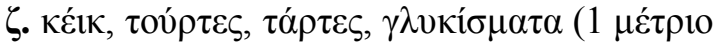
$\kappa о \mu \mu \alpha ́ \tau \imath)$

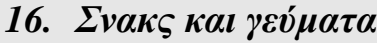

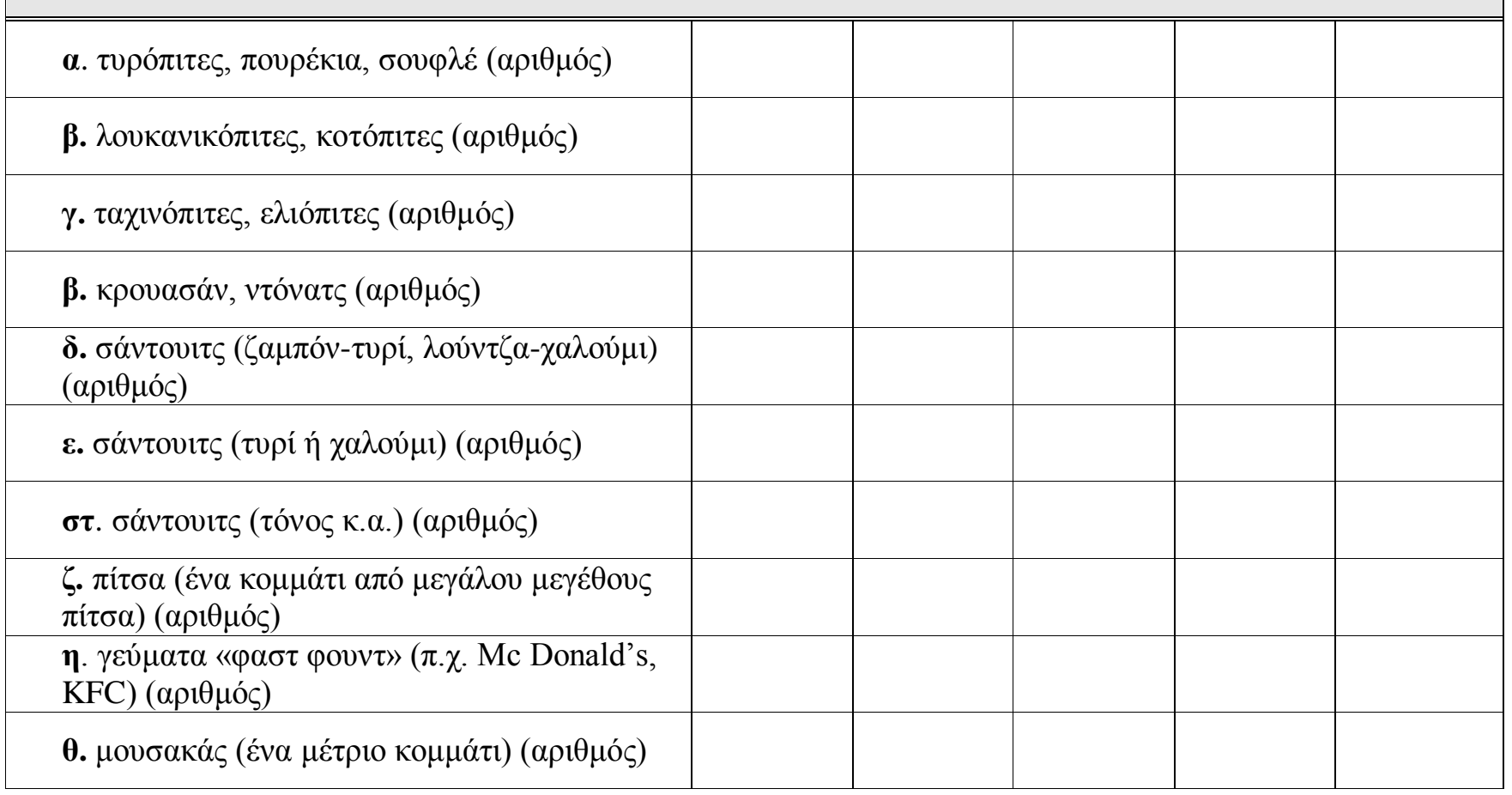




\begin{tabular}{|c|c|c|c|c|c|}
\hline \multirow[b]{2}{*}{ ТРОФІМА } & \multicolumn{5}{|c|}{ ¿YXNOTHTA } \\
\hline & Поте́ & 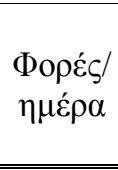 & $\begin{array}{c}\text { Форе́́/ } \\
\varepsilon \beta \delta о \mu \alpha ́ \delta \alpha\end{array}$ & $\begin{array}{l}\text { Форе́ } / / \\
\mu \eta ் v \alpha\end{array}$ & $\begin{array}{c}\Delta \varepsilon v \\
\theta v \mu \alpha ́ \mu \alpha / \\
\Delta \varepsilon v \\
\gamma \nu \omega \rho i ́ \zeta \omega\end{array}$ \\
\hline \multicolumn{6}{|l|}{ 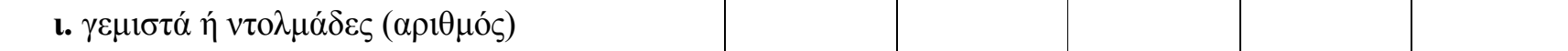 } \\
\hline \multicolumn{6}{|c|}{ 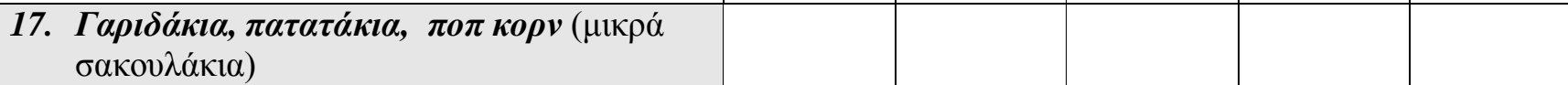 } \\
\hline \multicolumn{6}{|c|}{ 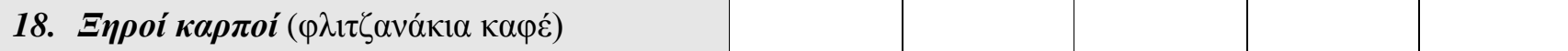 } \\
\hline \multicolumn{6}{|l|}{ 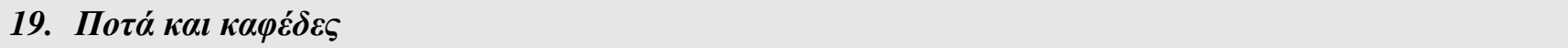 } \\
\hline \multicolumn{6}{|l|}{ 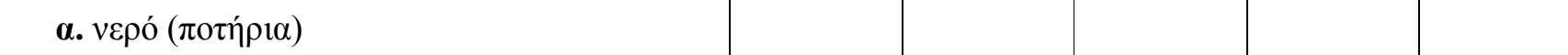 } \\
\hline \multicolumn{6}{|c|}{ 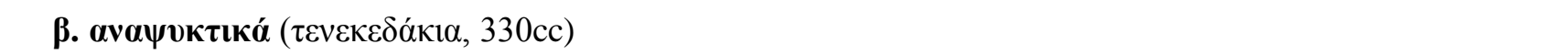 } \\
\hline \multicolumn{6}{|l|}{ 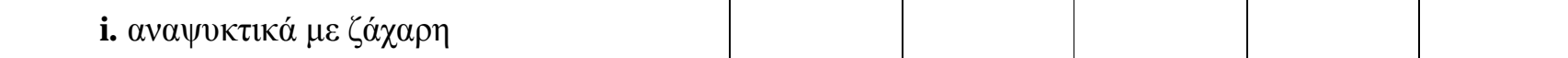 } \\
\hline \multicolumn{6}{|c|}{ 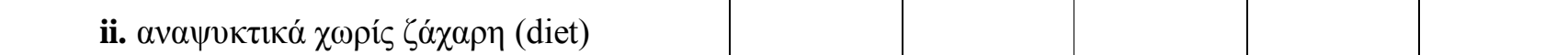 } \\
\hline \multicolumn{6}{|c|}{ 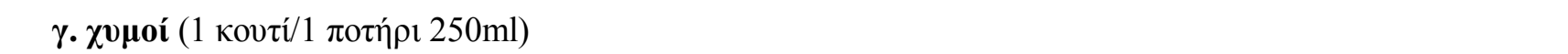 } \\
\hline \multicolumn{6}{|c|}{ 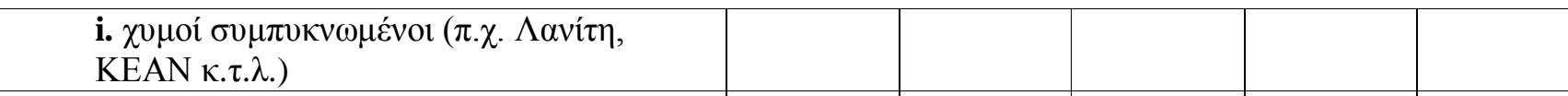 } \\
\hline \multicolumn{6}{|l|}{ 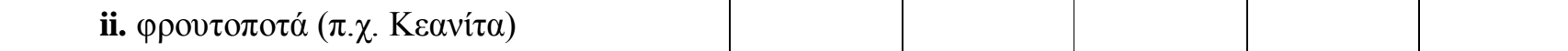 } \\
\hline \multicolumn{6}{|l|}{ 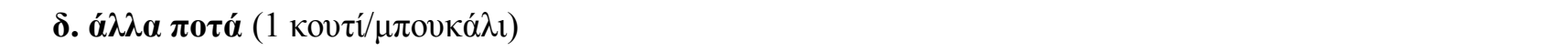 } \\
\hline \multicolumn{6}{|c|}{ 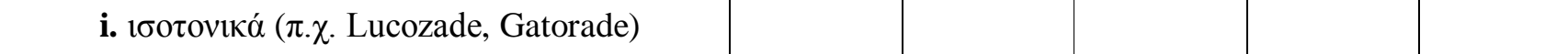 } \\
\hline \multicolumn{6}{|c|}{ ii. energy drinks ( $\pi \cdot \chi$. Redbull, Shark) } \\
\hline \multicolumn{6}{|l|}{ 20. $\Sigma v \mu \pi \lambda \eta \rho \omega ́ \mu \alpha \tau \alpha$} \\
\hline \multicolumn{6}{|c|}{ 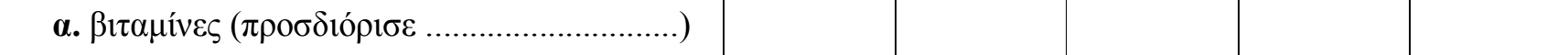 } \\
\hline \multicolumn{6}{|l|}{ 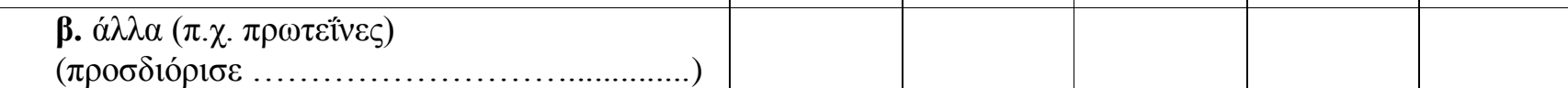 } \\
\hline \multicolumn{6}{|l|}{ 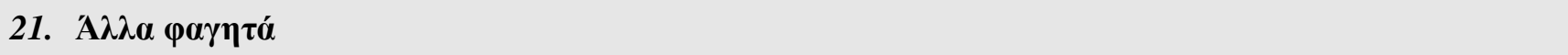 } \\
\hline \multicolumn{6}{|l|}{ Q. } \\
\hline В. & & & & & \\
\hline
\end{tabular}

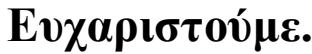




\section{EPSTHMATOЛOГIO}

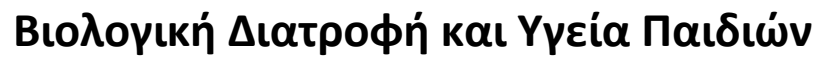

\begin{tabular}{|l|l|l|l|}
\hline Child's First and Last Name & \multicolumn{3}{|l|}{} \\
\hline $\begin{array}{l}\text { Parent's First and Last } \\
\text { Name }\end{array}$ & \multicolumn{2}{|l|}{} \\
\hline Primary Telephone & & Date & \\
\hline Questionnaire ID & & & \\
\hline
\end{tabular}

\section{PART A}

Please answer the following questions:

1. Education level:

Primary Education

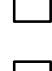

Secondary Education

University/College

Master's/Doctorate's

Other.

2. Mother's Occupation:

3. Father's Occupation

4. City/Village of Residence

5. ZIP Code:

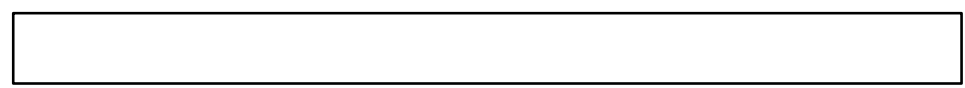

6. Length of Stay in Current Residence:

months/years 
7. How frequently have you used pesticides in your place of residence (including indoor and outdoor areas-garden, grass, lawn, trees) in the past 3 months? By pesticides, we refer to insect repellents, herbicides, insecticides, fungicides, or rat/mouse poisons.

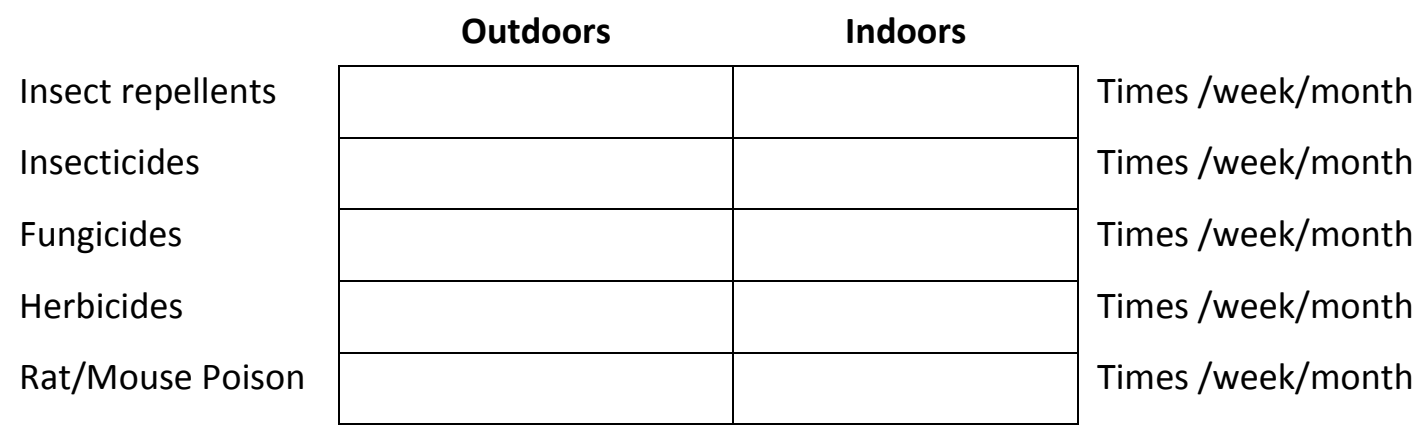

If you don't use pesticides, please continue at Part B.

8. When was the most recent use of pesticides in your place of residence? (days, weeks, months, etc.)?

\begin{tabular}{l|l|l|}
\multicolumn{1}{l}{} & \multicolumn{2}{c}{ Outdoors } \\
\cline { 2 - 3 } Insect repellents & & \\
\cline { 2 - 3 } Insecticides & & \\
\cline { 2 - 3 } Fungicides & & \\
\cline { 2 - 3 } Herbicides & & \\
\cline { 2 - 3 } Rat/Mouse Poison & & \\
\cline { 2 - 3 } & &
\end{tabular}

9. Do you know which pesticides are usually used in your place of residence? Please list them:

\section{Part B}

Please answer the following questions with regards to your child participating in the study:

1. Child's Gender: Male $\square$ Female

2. Child's Birthday:

3. Does your child have the tendency to put their fingers in their mouth (nail biting, finger/thumb sucking, etc.)?

Yes

No

4. How much time does your child spend in gardens or parks on a weekly basis?
Garden:
hours/week
Park:
hours/week 
5. How many hours per week does your child spend doing physical activities or sports on a weekly basis?

\begin{tabular}{|l|l|}
\hline & Hours/week \\
\hline Running & \\
\hline Cycling & \\
\hline Basketball & \\
\hline Football/Soccer & \\
\hline Volleyball & \\
\hline Swimming & \\
\hline Dance & \\
\hline $\begin{array}{l}\text { Other } \\
\text {.......................... }\end{array}$ & \\
\hline
\end{tabular}

6. How much time does your child spend in passive activities on a weekly basis?

\begin{tabular}{|l|l|}
\hline & Hours/week \\
\hline Television & \\
\hline Computer & \\
\hline Tablet & \\
\hline Mobile phone & \\
\hline $\begin{array}{l}\text { Other } \\
\text {............................. }\end{array}$ \\
\hline
\end{tabular}

\section{Part C (Completed by a Research Member)}

\begin{tabular}{|l|l|l|}
\hline Date & Starting Date $(. . . / . . . / \ldots . .)$. & Final Date (..............) \\
\hline Weight & & \\
\hline Height & & \\
\hline Waist circumference & & \\
\hline
\end{tabular}




\section{EPSTHMATOЛOГIO}

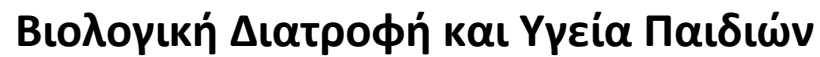

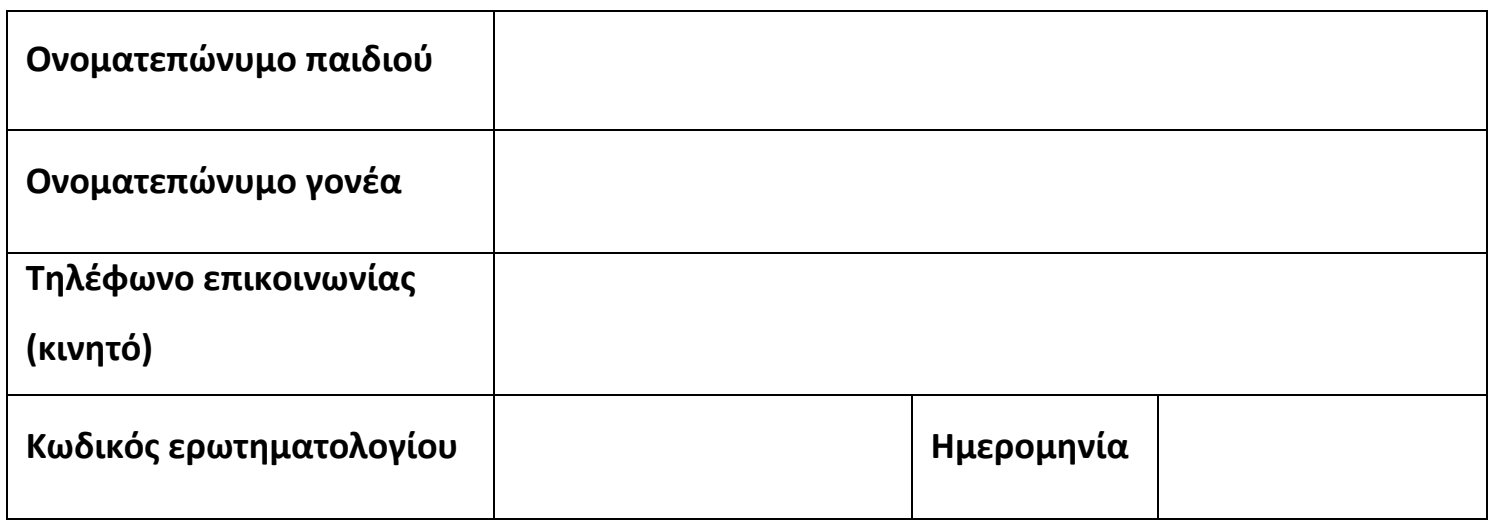

\section{Mépos A}

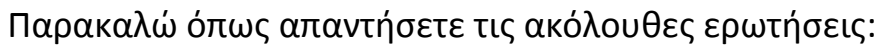

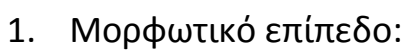

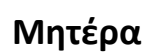

$\Delta$ пиоткко́

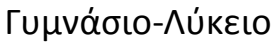

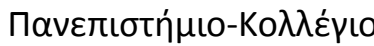

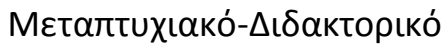

'A $\lambda \lambda_{0}$

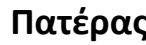

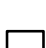

$\square$

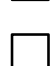




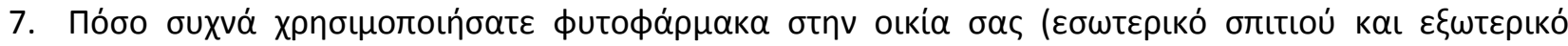

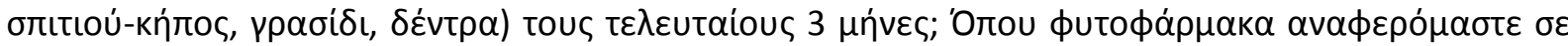

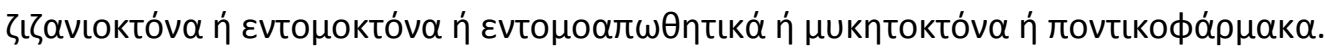

\begin{tabular}{|c|c|c|c|}
\hline \multirow[b]{2}{*}{ 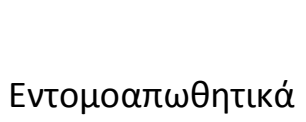 } & 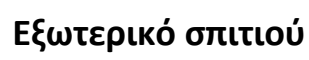 & 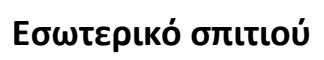 & \multirow[b]{2}{*}{ 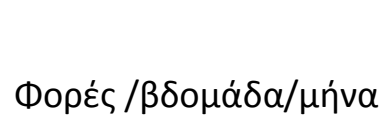 } \\
\hline & & & \\
\hline Evтоноктóv $\alpha$ & & & 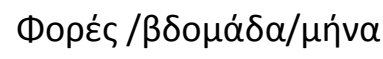 \\
\hline Мuкптоктóva & & & 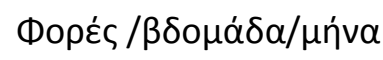 \\
\hline 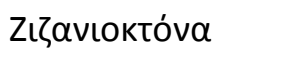 & & & 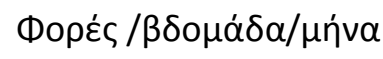 \\
\hline 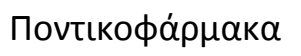 & & & 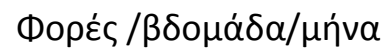 \\
\hline
\end{tabular}

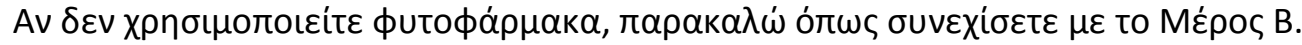

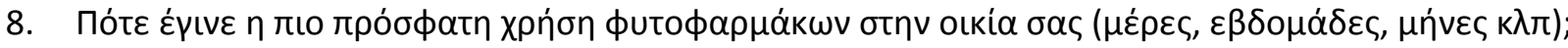

\begin{tabular}{|c|c|c|}
\hline & 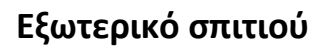 & 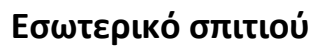 \\
\hline \multicolumn{3}{|l|}{ 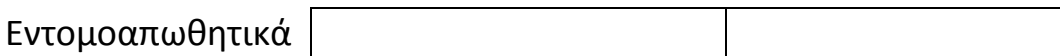 } \\
\hline \multicolumn{3}{|l|}{ Evтоноктóva } \\
\hline \multicolumn{3}{|l|}{ Мuкптокто́v $\alpha$} \\
\hline \multicolumn{3}{|l|}{ 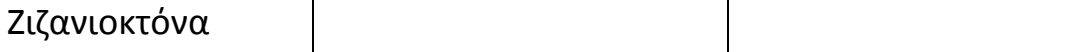 } \\
\hline 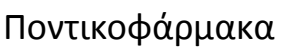 & & \\
\hline
\end{tabular}

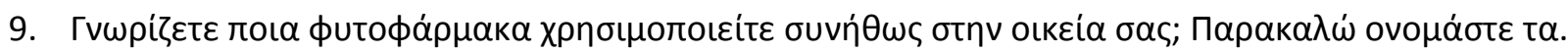

\section{Mépos B}

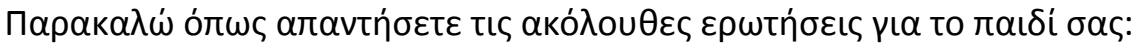

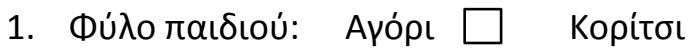

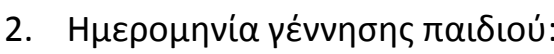

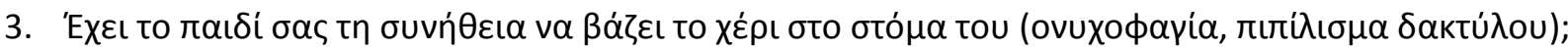
$\mathrm{Nal}$
'OXı

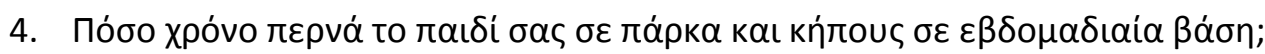
Ки́rос:

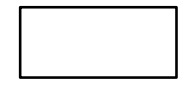
$\dot{\omega} \rho \varepsilon \varsigma / \varepsilon \beta \delta о \mu \alpha \dot{\delta} \alpha$
Па́рко:
$\dot{\omega} \rho \varepsilon \varsigma / \varepsilon \beta \delta о \mu \alpha \dot{\delta} \alpha$ 


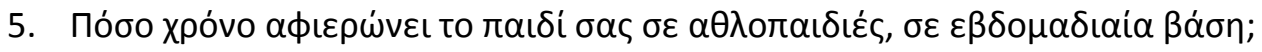

\begin{tabular}{|c|c|}
\hline & ' $\Omega \rho \varepsilon \varsigma / \varepsilon \beta \delta о \mu \alpha ́ \delta \alpha$ \\
\hline 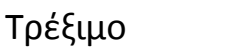 & \\
\hline 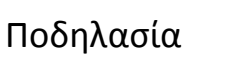 & \\
\hline 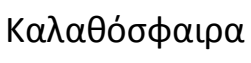 & \\
\hline 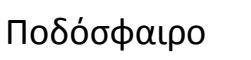 & \\
\hline 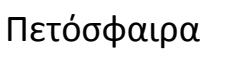 & \\
\hline 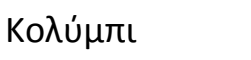 & \\
\hline Xopós & \\
\hline$A \lambda \lambda o$ & \\
\hline
\end{tabular}

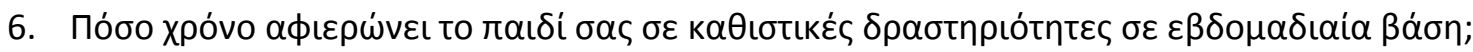

\begin{tabular}{|c|c|}
\hline & 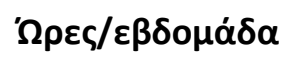 \\
\hline 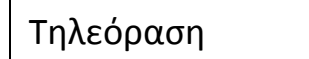 & \\
\hline Үло入оүьбти́s & \\
\hline Tablet & \\
\hline Kıvпtó & \\
\hline 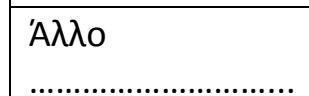 & \\
\hline
\end{tabular}

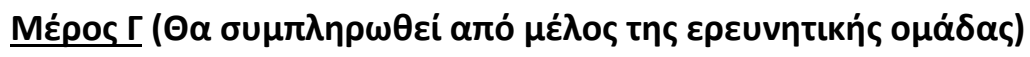

\begin{tabular}{|c|c|c|}
\hline 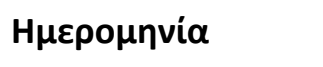 & 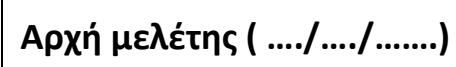 & 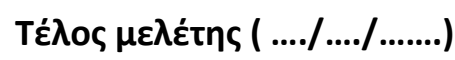 \\
\hline Bópos & & \\
\hline 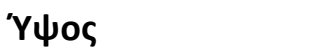 & & \\
\hline 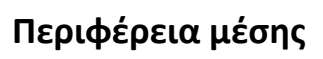 & & \\
\hline
\end{tabular}

